CASE REPORT

\title{
AN UNUSUALLY LATE PRESENTATION OF MALIGNANCY IN A PATIENT WITH GARDNER SYNDROME
}

S. Sopan ${ }^{1}$, E.K.D.M. Wickramaratne ${ }^{2}$, H.M.S.R.B. Kotakadeniya ${ }^{3}$, S. Wijetunge ${ }^{2}$

${ }^{1}$ Teaching Hospital, Peradeniya, Sri Lanka

${ }^{2}$ Department of Pathology, Faculty of Medicine, University of Peradeniya, Sri Lanka

${ }^{3}$ Department of Surgery, Faculty of Medicine, University of Peradeniya, Sri Lanka

Corresponding Author: Dr. S. Sopan; Email: siva.sopan@ gmail.com

\begin{abstract}
A 64-year-old male who presented with bleeding per rectum and altered bowel habits for 6 months was found to have numerous polyps in the colon during colonoscopy. He also had two osteomas on the forehead and several epidermal cysts in the scrotal skin. His brother had a history suggestive of a similar disease. The patient underwent total proctocolectomy and ileal pouch - anal anastomosis. Pathological examination of the resected specimen revealed an adenocarcinoma in addition to numerous adenomata. The patient was diagnosed to have colonic adenocarcinoma complicating Gardner syndrome.
\end{abstract}

Key words: Osteomas, Epidermal cysts, Adenomatous polyposis syndromes, Gardner syndrome.

\section{Introduction}

Gardner syndrome (GS) is a rare autosomal dominant inherited disorder ${ }^{1}$ with a high degree of penetrance. It is characterized by the presence of numerous intestinal polyps (more than 100) with extra-intestinal manifestations of bone and soft-tissue such as desmoid fibromatosis, lipomas, osteomas and epidermal cysts. This is regarded as a clinical subgroup of familial adenomatous polyposis (FAP) and may present at any age from 2 months to 70 years with colonic and extra colonic symptoms ${ }^{2}$. Patients with FAP and its variants have an invariable life time risk of one or more intestinal polyps transforming into invasive malignancy at a relatively younger age. GS cannot be separated from FAP when considering studies that describe its overall prevalence. Estimates for the prevalence of the combined syndromes vary from 1 in 6,850 to 1 in 31,250 (2.29 to 3.2 cases per 100,000 persons $)^{3-5}$. Prevalence appears fairly constant throughout the world with men and women affected equally. Twenty to 30 percent of newly diagnosed cases, i.e. those who do not belong to previously identified families, appear to represent new mutations. New cases may also arise from mosaic inheritance, which implies that a mutation has occurred in parents' sperm or ova, but not in other cells of the body, so the parent did not have clinical disease $^{6}$. Data for prevalence in Sri Lanka is not available. We present here a case of Gardner syndrome presenting with malignancy at an unusually advanced age.

\section{Case Report}

A 64-year-old male presented with bleeding per rectum and altered bowel habits with a 
sense of incomplete evacuation and loss of appetite for the last 6 months. His brother had a similar disease and had undergone a bowel surgery in his fifties. He had hypertension and was an ex-smoker and has consumed alcohol in the past. On examination, he was emaciated. There were two bony lumps over the forehead. His blood pressure was 180/120 mmHg. Abdominal and rectal examination were normal. The scrotum showed several epidermal cysts.

He was anaemic with a haemoglobin level of $8.1 \mathrm{~g} / \mathrm{dl}$. Abdominal ultrasound scan did not reveal any intra-abdominal masses. X-ray of the skull showed osteomas over the frontal bone (Figure 1).

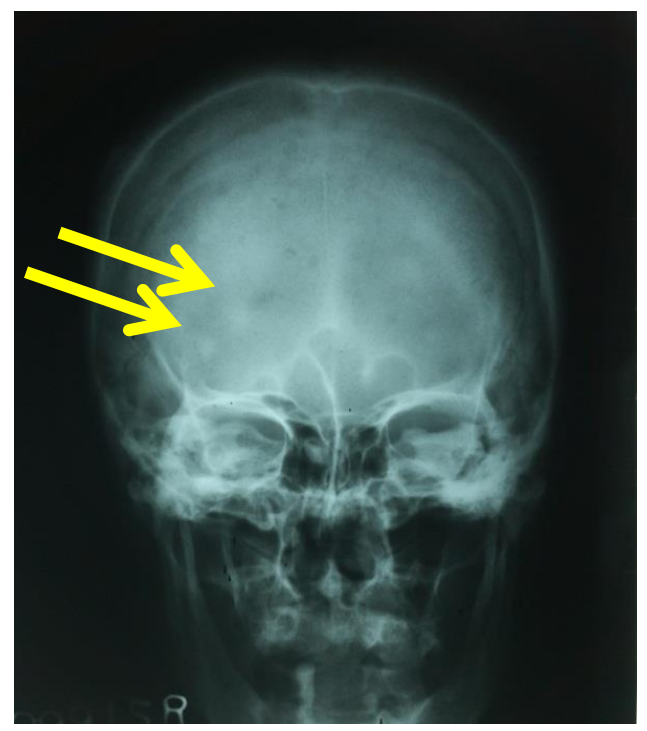

Figure 1. The skull $X$ ray showing osteomas over the frontal bone (Arrows)

Colonoscopy showed multiple (more than 100) polyps of varying sizes throughout the colon. No malignant appearing masses were observed. Based on these findings a working diagnosis of a variant of familial adenomatous polyposis - Gardner syndrome was made.
Total proctocolectomy and ileal pouch - anal anastomosis with de-functioning ileostomy was performed. Gross pathological examination of the colectomy specimen revealed numerous polyps of varying sizes (Figure 2).

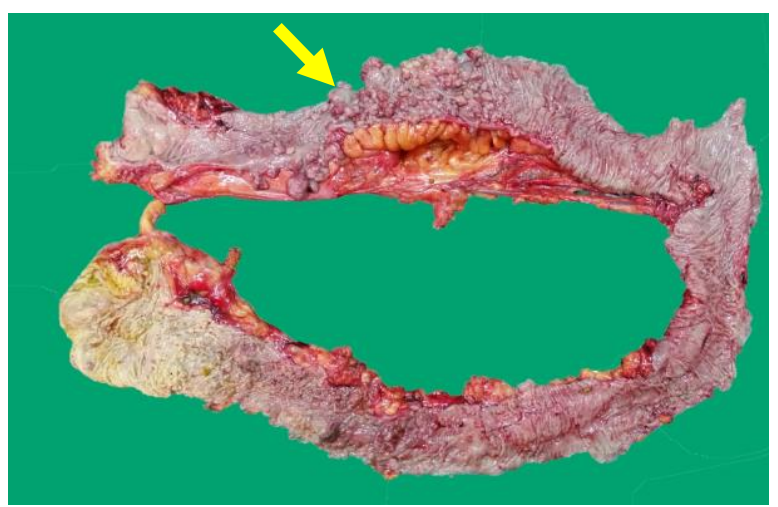

Figure 2. Colon with polyps of varying sizes. The arrow indicates the area where the malignant tumour was found.

The largest polyp was pedunculated and was $25 \mathrm{~mm}$ in diameter. The rest of the polyps were both pedunculated and sessile and the sizes ranged from $25 \mathrm{~mm}$ to less than $1 \mathrm{~mm}$ in diameter. A firm tumour was noted as a thickening of the wall in the sigmoid colon. The mucosa over the tumour was flattened (Figure 2).

Microscopic examination through the tumour showed a moderately differentiated adenocarcinoma, infiltrating the entire thickness of the colonic wall but not extending into the subserosal adipose tissue. The tumour did not appear to be arising from a polyp. Two pericolic lymph nodes showed tumour deposits. The TNM stage was T2N1M0. The polyps were villous, tubulovillous (Figure 3) and tubular adenomata with low grade dysplasia. There were oligocryptal and flat adenomata as well. 


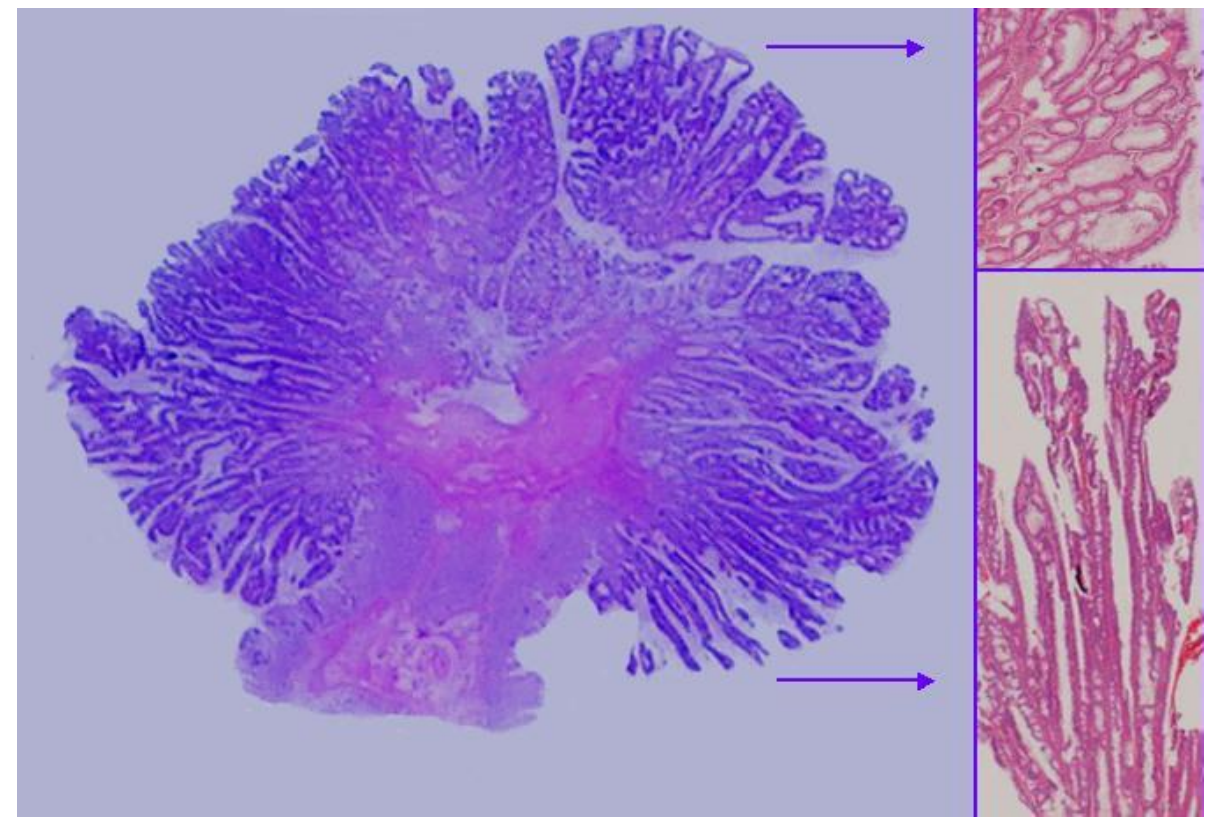

Figure 3. A tubulovillous adenoma (H \& E whole mount view). Insets: the lower box shows villous structures and the upper box shows tubular structures (H \& E X4).

The patient was referred to an oncologist and the reversal of the ileostomy was planned after 6-8 weeks. The family members are also going to be screened.

\section{Discussion}

Patients with FAP and its variants have a $100 \%$ lifetime risk of one or more of the polyps developing in to an adenocarcinoma through adenoma - carcinoma sequence and they develop carcinoma earlier than in sporadic cases, usually at around 40 years ${ }^{10}$. Therefore, the late presentation with malignancy in this patient is somewhat unusual. Furthermore, the malignancy in this patient did not appear to be arising from a polyp and the luminal surface of the malignancy was flattened. The entire spectrum of adenoma from microscopic monocryptal and oligocryptal to polypoid adenomata and flat adenomata can be demonstrated in patients with FAP. Usually high grade dysplasia and subsequent invasive malignancy is associated with larger polypoid adenomata. Infrequently flat adenomata show malignant transformation. Therefore, it is possible that this malignancy developed in a flat adenoma. Furthermore, the tumour had predominantly outward growth with only subtle mucosal changes which may have led to unrecognition with colonoscopy.

It was reported that Gardner syndrome is caused by truncating mutations of the APC gene (codons 1403 and 1578) differing from classic FAP (codons 169-1600) and attenuated FAP (amino terminal to codon $157)^{2}$. However, there is evidence that patients with identical mutations may have different phenotypic expressions because of unclear reasons ${ }^{11}$. The unusual features observed in this patient could be due to a variation from the typical genetic mutation. We were unable to perform the genetic testing for APC mutation in this patient. 
The majority of patients with FAP and its variants have a family history, however, about $25 \%$ can present without a family history due to a new dominant mutation. In the present case, presence of an affected brother indicates inheritance of a germ line mutation. In FAP, screening of the family members and prophylactic colectomy for those who are affected are indicated. However, extracolonic manifestations such as desmoid tumors of the mesenteric and abdominal wall can develop even after colectomy ${ }^{1}$.

The total proctocolectomy in combination with ileal pouch - anal anastomosis is the best surgical procedure for patients with FAP and its variants. This procedure has the advantage of removal of the entire large intestine mucosa, thus preventing the possible carcinogenesis and avoiding a permanent stoma $^{12}$.

There were four cases on variants (Cribriform morular and columnar variant) of Papillary Thyroid cancer and a study on Congenital hypertrophy of retinal pigment epithelium (CHRPE) in patients with FAP reported from Sri Lanka ${ }^{7-9}$. A study on uses of familial adenomatous polyposis registry also had been published ${ }^{14}$.

Though our patient had a positive family history (His brother had colectomy at another hospital, details were not available) he did not undergo screening. A national Polyposis Registry with a system to screen (Genetic study and Colonoscopy) the family is needed in Sri Lanka. It will allow the family members of the patients to have prophylactic colectomy before cancer sets in.

\section{Conclusion}

FAP or its variants may present at older age with late development of cancer. A polyposis registry and a family screening system is needed to improve the outcome of patients with FAP and its variants.

\section{References}

1. Eldon J. Gardner and Ralph C. Richards. Multiple cutaneous and subcutaneous lesions occurring simultaneously with hereditary polyposis and osteomatosis. American Journal of Human Genetics 1953; 5(2): 139-147.

2. Guo-Li Gu, Shi-Lin Wang, Xue-Ming Wei, Li Bai. Diagnosis and treatment of Gardner syndrome with gastric polyposis: A case report and review of the literature. World Journal of Gastroenterology 2008; 14(13): 2121-212.

3. Bussey HJR. Familial polyposis coli. In: Family Studies, Histopathology, Differential Diagnosis and Results of Treatment, Johns Hopkins University Press, Baltimore 1975.

4. Järvinen HJ Epidemiology of familial adenomatous polyposis in Finland: impact of family screening on the colorectal cancer rate and survival. Gut 1992; 33(3):357.

5. Bülow S, Faurschou Nielsen T, Bülow C, Bisgaard ML, Karlsen L, Moesgaard F The incidence rate of familial adenomatous polyposis. Results from the Danish Polyposis Register. Int J Colorectal Dis 1996;11(2):88.

6. Aretz S, Stienen D, Friedrichs N, Stemmler S, Uhlhaas S, Rahner N, Propping P, Friedl W Somatic APC mosaicism: a frequent cause of familial adenomatous polyposis (FAP). Hum Mutat 2007;28(10):985.

7. Liyanapathirana N, Seneviratne SA, Samarasekera A distinct variant of papillary thyroid carcinoma indicating familial adenomatous polyposis (FAP): a case report and brief review BMC Res Notes 2015; 8: 795. 
8. Nusliha A, Fernando F R, Deen K I, Dalpatadu K U A, Aruna Prasanna WL A D, Familial adenomatous polyposis (FAP) and papillary thyroid cancer: A rare form of familial cancer syndrome The Sri Lanka Journal of Surgery 2012; 30(1): 34-35.

9. Pradeep I.H.D.S, Chandrasingha P.C, Kannangara K.R, Epitakaduwa E.K.G.D.C, Fernando R.F. Columnar cell variant of papillary thyroid carcinoma - a rare and clinically aggressive variant. The Sri Lanka Journal of Surgery 2015; 33(2): 44-45.

10. Jerrold R. Turner. Adenomatous Polyposis. In: Vinay Kumar, Abul K. Abbas, Jon C. Aster. Robbins and Cotran. Pathologic Basis of Disease. Canada: Elsevier Saunders, 2015: 809815.

11. Jonathan Butler, Claire Healy, Mary Toner,Stephen Flint Gardner syndrome-review and report of a case. Oral Oncology 2005; 41(5): 89-92.

12. Nandakumar G, Morgan JA, Silverberg D, Steinhagen RM. Familial polyposis coli: clinical manifestations, evaluation, management and treatment. Mount Sinai Journal of Medicine 2004; 71: 384-391.

13. Nusliha A, Dalpatadu U, Amarasinghe B, Chandrasinghe PC, Deen KI. Congenital hypertrophy of retinal pigment epithelium (CHRPE) in patients with familial adenomatous polyposis (FAP); a polyposis registry experience BMC Research Notes 2014; 7:734.

14. Dalpatadu1 KUA, Anwar N, Wijesuriya SR E, Kumarage SK, Amarasinghe B, Deen KI. Uses of a familial adenomatous polyposis registry. Ceylon Medical Journal 2011; 56: 66-69. 